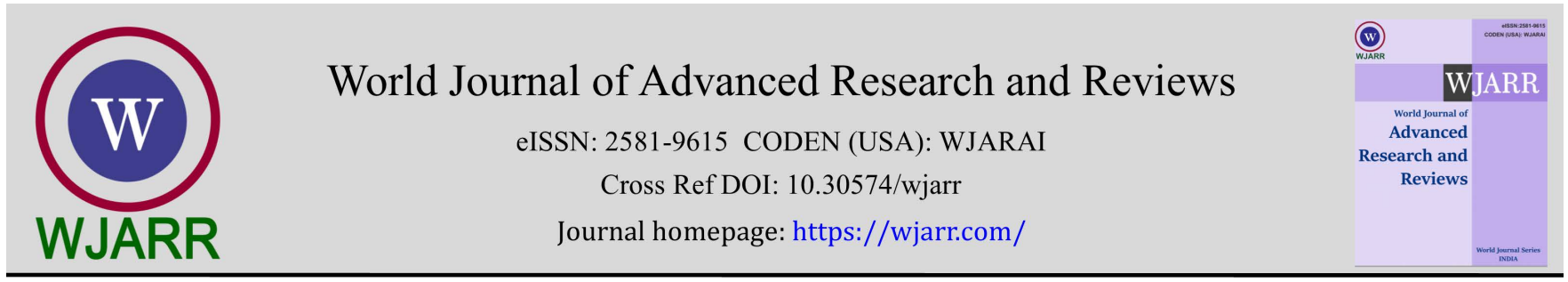

(REVIEW ARTICLE)

Check for updates

\title{
Microsphere as a novel strategy for the long-term use of corticosteroids in asthma patients during the COVID-19 Pandemic
}

\author{
Visuddho ${ }^{1,}{ }^{*}$, Nabil Alfin Juhri ${ }^{1}$, Ludy Diana Wiradhika ${ }^{1}$ and Reny I'tishom ${ }^{2, *}$ \\ ${ }^{1}$ Faculty of Medicine, Universitas Airlangga, Surabaya, Indonesia. \\ 2 Department of Biomedical Sciences, Faculty of Medicine, Universitas Airlangga, Surabaya, Indonesia.
}

World Journal of Advanced Research and Reviews, 2022, 13(01), 468-472

Publication history: Received on 06 December 2021; revised on 10 January 2022; accepted on 12 January 2022

Article DOI: https://doi.org/10.30574/wjarr.2022.13.1.0020

\begin{abstract}
The use of corticosteroids during the (Coronavirus Disease 2019) COVID-19 has become a new concern. Corticosteroids are the main therapy in asthmatic patients with anti-inflammatory and immunosuppressant effects. Patients with the need for long-term use of corticosteroids, such as asthma patients, are aware of the risk from infection obtained by the side effect of long-term corticosteroid use. A Recent study has innovated a new drug delivery agent, called Microsphere that can specify the targeted therapy. This new therapy may potentially increase the treatment efficacy with the lesser side effects. Here we review a study that uses the Microsphere for delivering corticosteroids. Additional studies are warranted to support the use of Microspheres as novel drug delivery for asthma patients during the COVID-19 pandemic.
\end{abstract}

Keywords: Asthma; Corticosteroids; COVID-19; Microsphere; Therapy

\section{Introduction}

COVID-19 has become a global concern, with the number of cases reaching almost 269 million confirmed cases and 5.3 million deaths until December 2021 [1]. Patients with COVID-19 are associated with the need for medical services. In severe patients, a corticosteroid is frequently prescribed to treat hyper inflammation and ARDS, it has been found to reduce the patient's short-term mortality and decrease the need for a ventilator. The proper use of corticosteroids can improve or reduce inflammation due to COVID-19, but improper use will be dangerously related to various side effects such as being more susceptible to infection due to immunosuppression and hypokalemia [2, 3].

Corticosteroids are the primary therapy in asthmatic patients, and their use is expected to accelerate symptom improvement and reduce the likelihood of recurrence in acute asthma exacerbations [4]. The use of corticosteroids for asthmatic patients during the pandemic has received particular attention due to their side effects, such as being susceptible to infection due to the immunosuppressive effect [5]. The side effects that are quite detrimental and potentially dangerous have made several recent studies focused on targeting specific corticosteroid targets to increase the efficacy of therapy and reduce the side effects.

Specific targets in treatment can increase effectiveness, thereby reducing the risk of side effects and increasing efficacy [6]. Microsphere acts on specific targets of particular concern, which are in the form of small particles ranging in size from 1 to $1000 \mathrm{~m}$ and in the size of 5 to $15 \mathrm{~m}$ which the pulmonary capillary network can directly trap, which are specific to the lungs [6].

\footnotetext{
* Corresponding author: Reny I'tishom

Department of Biomedical Sciences, Faculty of Medicine, Universitas Airlangga, Surabaya, Indonesia.

Copyright $(2022$ Author(s) retain the copyright of this article. This article is published under the terms of the Creative Commons Attribution Liscense 4.0.
} 
Microsphere can help achieve optimal drug concentrations without increasing unwanted systemic effects so that can reduce the risk of side effects especially for immunosuppressed patients during the COVID-19 pandemic [6]. One of the Microsphere's indications is the usage of Microsphere as an introductory poly-lactic-co-glycolic acid (PLGA) for budesonide in asthma therapy. A study using a Microsphere (PLGA) as an introduction to budesonide showed an increase in the efficiency of the regimen [7]. Therefore, we aim to discuss the potential use of Microspheres as a practical introduction to budesonide in immunosuppressive therapy during the COVID-19 pandemic.

\section{Material and methods}

This review was conducted by thoroughly searching several databases, including PubMed, ScienceDirect, Google Scholar, Scopus, and other websites. For the search strategy, we used keywords "Microsphere," "Corticosteroids," "COVID-19", "Asthma," and its synonyms to find all relevant related articles. Boolean operators ("OR," "AND," "NOT") were used to get more specific results. The search was limited to articles in the English language only. We also use the information of COVID-19 from the World Health Organization website.

\section{Results}

\subsection{The corticosteroid used for antiinflammation therapy}

Corticosteroids have potent anti-inflammatory and immunosuppressant effects, so they are often used in various therapies. Patients who require long-term corticosteroids can generally be divided into patients with primary, secondary, or tertiary adrenal insufficiency and patients who require potent anti-inflammatory and immunosuppressant effects [8]. A systematic literature review found that corticosteroids are mostly used to treat autoimmune, asthma, chronic obstructive pulmonary disease (COPD), and other lung diseases [5]. The use of oral corticosteroids has also been found to have an essential role in the treatment of chronic rhinosinusitis. However, the dose and frequency of use need special attention to prevent side effects such as suppression of the adenoids, growth suppression, or increased intraocular pressure [9].

Long-term use of corticosteroids is also required in patients with lung transplantation. This usage is expected to prevent organ rejection from the body. Long-term use of corticosteroids in these patients has the consequence of infection and malignancy due to its immunosuppressive effect [10]. Studies in asthmatic patients have shown that higher doses of long-term oral corticosteroids have a higher risk of developing complications [11]. This is due to the high systemic exposure to corticosteroids.

Although the dosage recommendations for long-term use of corticosteroids have been lowered over the last few decades, they are still not a practical solution in reducing the incidence of side effects of therapy [5]. A cohort study found that users of oral corticosteroids were three times more likely to develop pneumonia and opportunistic infections than non-users of oral corticosteroids [12]. Even exposure to low-dose oral corticosteroids also increases the risk of infection [12].

\subsection{The corticosteroid used in COVID-19 pandemic}

The risk of infection using corticosteroids has become an essential topic of discussion during the Coronavirus Disease 2019 (COVID-19) pandemic. On January 7, 2020, the etiologic agent of COVID-19, Severe Acute Respiratory Syndrome Coronavirus 2 (SARS-CoV-2), was isolated from a group of patients with an outbreak of pneumonia in Wuhan, China (Zhou et al., 2020). This virus quickly spread to various countries with a high increase in cases. On November 25, 2020, COVID-19 cases in Indonesia reached 511,836 confirmed cases, with 16,225 deaths across 34 provinces [12]. The clinical spectrum of patients is quite broad, ranging from asymptomatic to severe pneumonia that can cause death. COVID-19 patients are also often associated with intensive care unit (ICU) admission rates and high mortality rates [13]. This shows the importance of recognizing and preventing factors that can increase the risk of COVID-19.

Patients requiring long-term use of oral corticosteroids are more susceptible to severe complications in SARS-CoV-2 infection [8]. This is probably due to the effect of immunosuppressants that can increase the risk of infection. The Global Initiative for Asthma (GINA) also recommends continuing the use of inhaled corticosteroids for asthmatic patients during the pandemic but recommends using biologic therapy for patients with severe asthma to reduce the use of oral corticosteroids [14]. In addition, the use of corticosteroids suspected to be one of the causes of the increase in opportunistic fungal infections in the COVID-19 epidemic [15]. 
COPD patients taking inhaled corticosteroids were also found to have a higher risk of COVID-related death than those taking long-acting beta-agonists (LABA) and long-acting muscarinic antagonists (LAMA). Asthmatic patients taking high doses of inhaled corticosteroids were also found to have a higher risk of death than those taking only short-acting betaagonists (SABAs) [16]. In addition, the management of asthma exacerbations or COPD in the COVID-19 pandemic also needs to be considered. It is currently advised to avoid using a nebulizer while in the hospital to avoid transmission of COVID-19. This shows that inhaled corticosteroids are ineffective enough to avoid an increased risk in the COVID-19 pandemic.

\subsection{The corticosteroid used in Asthma}

One of the most commonly used corticosteroids is budesonide. Budesonide is a drug regimen that is often used to treat asthma patients. Like other glucocorticoid groups, budesonide is a molecule with high lipid solubility, so it can diffuse across the membrane into the cytoplasm and bind to the glucocorticoid receptor (GR) [5]. Budesonide has a high receptor affinity. When compared with reference dexamethasone with a value of 100 , budesonide displays an affinity for the glucocorticoid receptor with a value of 935 [5].

After binding to the glucocorticoid receptor, a conformational change occurs that causes the dissociation of the chaperone protein. This will cause the appearance of a signal to the nucleus, resulting in translocation of the glucocorticoid receptor-ligand complex into the nucleus. Glucocorticoid receptors are activated to form homodimers and attach to specific genomes in DNA sequences known as glucocorticoid response elements (GRE). This response will increase the transcription of anti-inflammatory proteins such as secretory leukoprotease inhibitor (SLPI), mitogenactivated protein kinase phosphatase-1 (MKP-1) and glucocorticoid-induced leucine zipper (GILZ), which can inhibit the pro-inflammatory activity of nuclear factor $-\kappa B(N F-\kappa B)$ and activator protein-1 (AP-1) $[17,18]$. In addition, the attachment of glucocorticoid receptors to NF-B receptors has an effective suppressive effect of suppressing proinflammatory agents [18].

The best way to reduce the side effects of using corticosteroids is to reduce the systemic effects of corticosteroids. One of these effects can be reduced by increasing the drug's working time in the lungs. The thin pulmonary membrane and high blood flow to the lungs cause the rapid absorption of most drugs, so this aspect is essential to assess the selectivity of the drug to the lung. One of the best strategies to increase the drug's working time in the lungs is to use a Microsphere that has the characteristics of slowly releasing the drug and can increase the drug's effectiveness in the lung[19].

\subsection{Using Microsphere to specify corticosteroid target}

Particle size has an essential influence on the biodistribution of drugs to have a specific target [3]. Microspheres are small spherical particles with size variations ranging from 1 to $1000 \mathrm{~m}$. Particles smaller than $5 \mathrm{~m}$ can migrate to other organs such as the liver, spleen, and blood, but particles 5 to $15 \mathrm{~m}$ in size are known to be well distributed and trapped in the pulmonary capillary network, thus displaying specific drug delivery capabilities to the lung organs [6].

Microsphere has been confirmed to be a good drug delivery agent in asthma therapy. Microspheres can help achieve optimal drug concentrations in the lungs. This increase has an important effect, where not all conventional drugs can to reach the required concentration in the lung without increasing the unwanted systemic effects [6]. These results are linear with a previous study, which found that Microspheres can increase drug release time in asthmatic patients [16].

The use of Microspheres to introduce budesonide can be an effective therapy in asthma patients during the COVID-19 pandemic. The use of this combination is able to reduce the number of doses needed to reach the maximum dose in the lungs, thereby reducing systemic side effects that can lower the body's immune system. A study using Microsphere polylactic-co-glycolic acid (PLGA) as an introduction to budesonide showed an increase in the efficiency of the regimen [20]. Budesonide was also released slowly over 24 hours of in vitro studies. In addition, it was found that there was an increase in the efficiency of taking drugs into the lungs compared to without using a Microsphere [21].

Budesonide with PLGA introduction was also found to decrease bronchial hyperresponsiveness. This is a clinical improvement in asthmatic patients [21]. Budesonide-PLGA therapy was also found to have ameliorative benefits in a preclinical study of mice with asthma [22]. This study supports developments in the delivery and control of drugs with pulmonary action targets.

Microsphere can be used as an effective introduction to budesonide in immunosuppressant therapy during the COVID19 pandemic. This is achieved through a practical and selective release mechanism in the lung. The limitation of this study is that most of the studies are preclinical studies with a limited number of studies. This study supports the conduct of further experimental and clinical therapy to add sufficient scientific evidence so that the potential of this combination 
therapy can be applied and benefit asthmatic patients who require immunosuppressant therapy in the time of an infectious disease pandemic.

\section{Conclusion}

Corticosteroids are widely used to treat asthma patients. However, long-term use of corticosteroids can risk various side effects in patients, including the occurrence of infection, malignancy, and severe complications in SARS-CoV-2 infection due to immunosuppressant effects. The use of Microspheres as an introduction to asthma therapy is an effective therapy for asthma patients during the COVID-19 pandemic because it can increase working time in the lungs to reduce the side effects of long-term corticosteroid consumption. In addition, Microspheres as asthma therapy can help to achieve optimal drug concentrations in the lungs.

\section{Compliance with ethical standards}

\section{Acknowledgments}

This research did not receive specific grants from funding agencies in the public, commercial, or not-for-profit sectors.

\section{Disclosure of conflict of interest}

The author declares no conflict of interest.

\section{References}

[1] WHO. COVID-19 weekly epidemiological update. World Heal Organization [Internet]. 2021; (58): 1-23.

[2] Paassen JV, Vos JS, Hoekstra EM, Neumann KMI, Boot PC, Arbous SM. Corticosteroid use in COVID-19 patients: a systematic review and meta-analysis on clinical outcomes. Crit Care [Internet]. 2020; 24(1): 1-22.

[3] Yang Z, Liu J, Zhou Y, Zhao X, Zhao Q, Liu J. The effect of corticosteroid treatment on patients with coronavirus infection: a systematic review and meta-analysis. J Infect. 2020; 81(1): e13-20.

[4] Alangari AA. Corticosteroids in the treatment of acute asthma. Ann Thorac Med. 2014; 9(4): 187-92.

[5] Rice JB, White AG, Scarpati LM, Wan G, Nelson WW. Long-term Systemic Corticosteroid Exposure: A Systematic Literature Review. Clin Ther [Internet]. 2017; 39(11): 2216-29.

[6] Sreeharsha N, Venugopala KN, Nair AB, Roopashree TS, Attimarad M, Hiremath JG, et al. An efficient, lungtargeted, drug-delivery system to treat asthma via microparticles. Drug Des Devel Ther. 2019; 13: 4389-403.

[7] Yeh HW, Chen DR. In vitro release profiles of PLGA core-shell composite particles loaded with theophylline and budesonide. Int J Pharm [Internet]. 2017; 528(1-2): 637-45.

[8] Almeida MQ, Mendonca BB. Adrenal insufficiency and glucocorticoid use during the COVID-19 pandemic. Clinics. 2020; 75: 1-3.

[9] Campbell RG. Risks and management of long-term corticosteroid use in chronic rhinosinusitis. Curr Opin Otolaryngol Head Neck Surg. 2018; 26(1): 1-7.

[10] Ivulich S, Dooley M, Kirkpatrick C, Snell G. Clinical Challenges of Tacrolimus for Maintenance Immunosuppression Post-Lung Transplantation. Transplant Proc [Internet]. 2017; 49(9): 2153-60.

[11] Dalal AA, Duh MS, Gozalo L, Robitaille MN, Albers F, Yancey S, et al. Dose-response relationship between longterm systemic corticosteroid use and related complications in patients with severe asthma. J Manag Care Spec Pharm. 2016; 22(7): 833-47.

[12] Zazzali JL, Broder MS, Omachi TA, Chang E, Sun GH, Raimundo K. Risk of corticosteroid-related adverse events in asthma patients with high oral corticosteroid use. Allergy Asthma Proc. 2015; 36(4): 268-74.

[13] Huang C, Wang Y, Li X, Ren L, Zhao J, Hu Y, et al. Clinical features of patients infected with 2019 novel coronavirus in Wuhan, China. Lancet. 2020; 395(10223): 497-506.

[14] GIFA. COVID-19 : GINA Answers to Frequently Asked Questions on asthma Management. 2020. 
[15] Salehi M, Ahmadikia K, Badali H, Khodavaisy S. Opportunistic Fungal Infections in the Epidemic Area of COVID19: A Clinical and Diagnostic Perspective from Iran. Mycopathologia [Internet]. 2020;185(4): 607-11.

[16] She W, Mei Z, Zhao H, Li G, Lin Y. Nebulized inhalation of anti-nerve growth factor Microspheres inhibits airway remodeling in an ovalbumin-induced rat asthma model. J Aerosol Med Pulm Drug Deliv. 2019; 32(2): 70-7.

[17] Barnes PJ. Corticosteroid effects on cell signalling. Eur Respir J. 2006; 27(2): 413-26.

[18] Pelaia G, Vatrella A, Busceti MT, Fabiano F, Terracciano R, Matera MG, et al. Molecular and cellular mechanisms underlying the therapeutic effects of budesonide in asthma. Pulm Pharmacol Ther [Internet]. 2016; 40: 15-21.

[19] Hochhaus G. New developments in corticosteroids. Proc Am Thorac Soc. 2004; 1(3): 269-74.

[20] Yeh HW, Chen DR. In vitro release profiles of PLGA core-shell composite particles loaded with theophylline and budesonide. Int J Pharm. 2017; 528(1-2): 637-45.

[21] Oh YJ, Lee J, Seo JY, Rhim T, Kim SH, Yoon HJ, et al. Preparation of budesonide-loaded porous PLGA microparticles and their therapeutic efficacy in a murine asthma model. J Control Release. 2011; 150(1): 56-62.

[22] Li J, Zheng H, Qin L, Xu EY, Yang L, Zhang L, et al. In vitro-in vivo correlation of inhalable budesonide-loaded large porous particles for sustained treatment regimen of asthma. Acta Biomater. 2019; 96: 505-16. 